\title{
Anti-hyperlipidemic Effects of Red Ginseng Acidic Polysaccharide from Korean Red Ginseng
}

\author{
Yi-Seong KwaK, ${ }^{a}$ Jong-Soo Kyung, ${ }^{a}$ Jong Soo Kim, ${ }^{b}$ Jae Youl Cho, ${ }^{*, c}$ and Man-Hee RheE ${ }^{d}$ \\ ${ }^{a}$ Korea Ginseng Corporation Central Research Institute; Daejon 305-805, Korea: ${ }^{b}$ KFDA; Seoul 122-704, Korea: \\ ${ }^{c}$ School of Bioscience and Biotechnology, and Institute of Bioscience and Biotechnology, Kangwon National University; \\ Chuncheon 200-701, Korea: and ${ }^{d}$ College of Veterinary Medicine, Kyungpook National University; Daegu 702-701, Korea. \\ Received October 6, 2009; accepted December 21, 2009; published online December 24, 2009
}

It has been reported that red ginseng acidic polysaccharide (RGAP), isolated from Korean red ginseng, displays immunostimulatory and anti-tumor activities. In a follow-up study, we have carried out a study on the antihyperlipidemic effects of RGAP using hyperlipidemic rats acutely induced by Triton WR1339 or corn oil intravenously injected. Oral administration of RGAP (100 to $1000 \mathrm{mg} / \mathrm{kg})$ dose-dependently reduced the serum levels of triglyceride (TG) up-regulated by Triton WR1339, an inducer of endogenous model hyperlipidemia. Moreover, RGAP treatment was shown to significantly decrease the levels of non-esterified fatty acid (NEFA) concomitant with TG reduction. However, such reduction effects were not observed in cases of total cholesterol (TC) and phospholipid levels increased under the same conditions, although there was an inhibitory tendency. Similar suppressive patterns were also seen in hepatic parameters (total lipids and TG) under the same conditions. The exogenous hyperlipidemic rat condition triggered by corn oil also supported the anti-hyperlipidemic activity of RGAP in serum and hepatic parameters of TG and NEFA. Interestingly, RGAP significantly enhanced the serum activity of lipoprotein lipase, a key hydrolytic enzyme of lipid molecules in lipoprotein, in a dose-dependent manner up to $\mathbf{8 0} \%$, implying potential involvement of this enzyme in lowering TG and NEFA by RGAP. Therefore, our data suggest that RGAP may play an additional role in reducing hyperlipidemic conditions, which can be used as a valuable neutraceutical application for the treatment of hyperlipidemia.

Key words Panax ginseng; red ginseng acidic polysaccharide; anti-hyperlipidemic effect

Korean ginseng (the root of Panax ginseng C. A. MEYER) has been known to be a valuable and important folk medicine in East Asian countries, including Korea, China, and Japan for about 2000 years. Panax ginseng is indeed being widely used to treat numerous diseases such as cancer, diabetes, and cardiovascular diseases. ${ }^{1)}$ Active constituents with curable features found in most of ginseng species include ginsenosides, polysaccharides, peptides, polyacetylenic alcohols, and fatty acids. ${ }^{2)}$ Of these, ginsenosides, a group of saponins with a triterpenoid dammarane structure, have been studied as major pharmacological components with a variety of effects such as anti-diabetic, anti-cancer, anti-inflammatory, antihyperlilidemic, and anti-atherosclerosis activities in ginseng. ${ }^{2-6)}$ Polysaccharide fractions from ginseng have also been explored to understand ginseng's biological roles in pharmacology in terms of immunostimulatory functions. ${ }^{2)}$ Numerous studies have revealed that polysaccharides from the root of Panax ginseng were known to have mitogenic activities, anti-tumor activities and immunostimulating activities in the cyclophosphamide-treated immunosuppressed mice. ${ }^{2,7-9)}$ It has also been reported that red ginseng acidic polysaccharide (RGAP) from Korean red ginseng was able to up-regulate immunostimulating and antitumor activities for activation of natural killer cells and nitric oxide production in macrophages and in tumor-bearing models. ${ }^{10,11)}$ Furthermore, acidic polysaccharides from ginseng root were found to reduce the incidence rate of benzo $[a]$ pyreneinduced autochthonous neoplasm. ${ }^{12)}$

In addition, the anti-metabolic disease effects of polysaccharide fractions have also been explored using in vitro and in vivo experimental models. It has been reported that nonsaponin fractions of Korean red ginseng, are capable of inhibiting epinephrine-induced lipolysis and of stimulating in- sulin-mediated lipogenesis from glucose in rat adipocytes. ${ }^{13)}$ Acidic polysaccharides from ginseng root were found to inhibit toxohormone L-induced lipolysis. ${ }^{13)}$ Acidic polysaccharides from Korean red ginseng modulated pancreatic lipase activity, and caused a reduction of plasma triglyceride levels after oral administration of corn oil emulsion to rats, implying the involvement of pancreatic lipase in the reduction of lipolysis. ${ }^{14)}$ Nonetheless, very few polysaccharides of RGAP are known to be effective against hyperlipidemia in vivo. Although several papers have indicated the role of polysaccharide fractions from ginseng play in this regard, the effect of RGAP on lipid metabolism and hyperlipidemic conditions has not been fully elucidated yet. In this study, therefore, we aimed to explore the anti-hyperlipidemic effect of orally administered RGAP from Korean red ginseng with hyperlipidemic rat models. To do this, both Triton WR1339-induced endogenous and corn oil-induced exogenous hyperlipidemic rat models were employed. It has been reported that Triton WR1339 treatment increases serum levels of total cholesterol (TC) and triglyceride (TG) by up-regulating 3-hydroxy-3methylglutaryl-coenzyme A (HMG-CoA) reductase activity and suppressing lipoprotein lipase (LPL) activity. ${ }^{15,16)}$ Moreover, corn oil treatment is also known to directly enhance serum parameters such as TG. ${ }^{17}$ ) Therefore, these models seem to be fully considered as proper hyperlipidemic models.

\section{MATERIALS AND METHODS}

Materials Korean red ginseng made by steaming and drying fresh ginseng root (Panax ginseng C. A. MEYeR) kindly supplied by the Korea Ginseng Corporation (Daejon, Korea). Triton WR1339 and gemfibrozil were purchased from Sigma Chemical Co. (St. Louis, MO, U.S.A.). Absolute 
alcohol and sulfuric acid were purchased from Fluka (Buchs, Switzerland). All other chemicals and reagents used were of sigma grade.

Preparation of RGAP RGAP was isolated from Korean red ginseng as described previously. ${ }^{18)}$ Briefly, Korean red ginseng powder was percolated with 5 volumes of $70 \%$ ethanol to extract ethanol-soluble materials. Remaining residues were then percolated with 5 volumes of distilled water and the resulting water-soluble fractions were concentrated by vacuum evaporation. The concentrates were ultrafiltrated to completely cut off small molecules less than $10 \mathrm{kDa}$ by Ultrafiltrater (Ultrafiltration kit, Millipore, Pellicon 2, Bedford, MA, U.S.A.) and to obtain the non-ultrafiltraed fraction consisting of acidic polysaccharide (yield: about $15 \%$ ) (Fig. 1A). The analysis of component sugars in RGAP by HPLC revealed that the acidic sugars in RGAP contained galacturonic acid as a major component and glucuronic acid as a minor component (Fig. 1B). One milligram of RGAP contained less than 0.006 EU of endotoxin. ${ }^{19)}$

Animals Male Sprague-Dawley rats weighing 185$200 \mathrm{~g}$ were purchased from Semtako (Osan, Korea) and were supplied with a commercial diet (Samyang, Korea) and water ad libitum. They were housed in a temperature-controlled room at $20^{\circ} \mathrm{C}$ with lighting 7 a.m. to 7 p.m. Humidity was maintained between $50-70 \%$. The animals received humane care in accordance with the Guide for the Care and Use of Laboratory Animals, published by the U.S. National Institute of Health (NIH Publication, revised in 1985).

Triton WR1339-Induced Endogenous Hyperlipidemic Model Endogenous hyperlipidemia was induced in the rats with Triton WR1339 as reported previously. ${ }^{20)}$ Briefly, sixty rats were randomized into six groups of normal, control, and RGAP groups (R100, R300, R500, and R1000) in order to evaluate the endogenous anti-hyperlipidemic effect. The RGAP groups were orally administered with RGAP dissolved in $1 \mathrm{ml}$ of D.W. at doses of 100, 300, 500 and 1000 $\mathrm{mg} / \mathrm{kg}$ body weight for $3 \mathrm{~d}$ to examine short-term preventive effect. After this period, all the animals were fasted for $12 \mathrm{~h}$ prior to treatment with Triton WR1339. Control and RGAP groups received a single dose of Triton WR1339 (150 $\mathrm{mg} / \mathrm{kg}$ ), injected into a tail vein of the rat $1 \mathrm{~h}$ after administration of RGAP. Blood was drawn by heart puncture $2 \mathrm{~h}$ after RGAP administration, based on previous paper, ${ }^{21)}$ and serum was obtained by centrifugation at $3000 \mathrm{rpm}$ for $15 \mathrm{~min}$.

Corn Oil-Induced Exogenous Hyperlipidemic Model in Rats Exogenous hyperlipidemia was induced in rats with corn oil as reported previously. ${ }^{22)}$ Briefly, to evaluate the exogenous anti-hyperlipidemic effect, 60 rats were randomized into six groups (NC, control, R100, R300, R500, and R1000). RGAP groups were orally administered doses of $100,300,500$ or $1000 \mathrm{mg} / \mathrm{kg}$, respectively, for $3 \mathrm{~d}$, based on previous report in which it was found that there was no side effect of RGAP treatment for 2 weeks up to $1 \mathrm{~g} / \mathrm{kg} .{ }^{23)}$ Diet and drinking water were given ad libitum. After $3 \mathrm{~d}$, all the animals were fasted for $12 \mathrm{~h}$. Control and RGAP groups were orally administered a single dose of corn oil $(2.0 \mathrm{~g} / \mathrm{g}) 2 \mathrm{~h}$ after treatment. After $2 \mathrm{~h}$, blood and serum were drawn and obtained using the same method dexcribed for Triton WR1339-treated animals.

Liver Sample Preparation Liver sample was prepared according to previous report. ${ }^{24)}$ Briefly, liver (1 g) was homogenized with $20 \mathrm{ml}$ of chloroform/methanol (2/1). After dispersion, the whole mixture was agitated during 15 $20 \mathrm{~min}$ in an orbital shaker at room temperature. The homogenate was either filtered or centrifuged to recover the liquid phase. The solvent was washed with water $(4 \mathrm{ml}$ for $20 \mathrm{ml}$ ). After vortexing few seconds, the mixture was centrifuged at low speed $(2000 \mathrm{rpm})$. After removing the upper phase, the lower chloroform phase containing lipids was used to determine lipid amount by evaporating under vacuum and drying condition at $50^{\circ} \mathrm{C}$ for $6 \mathrm{~h}$.

Biochemical Parameter Analysis Serum levels of TC, TG, high density lipoprotein-cholesterol (HDL-C), and nonesterified fatty acid (NEFA) were determined by Automatic analyzer (Hitachii 7020, Japan). TC, TG, and HDL-C in the
(A)

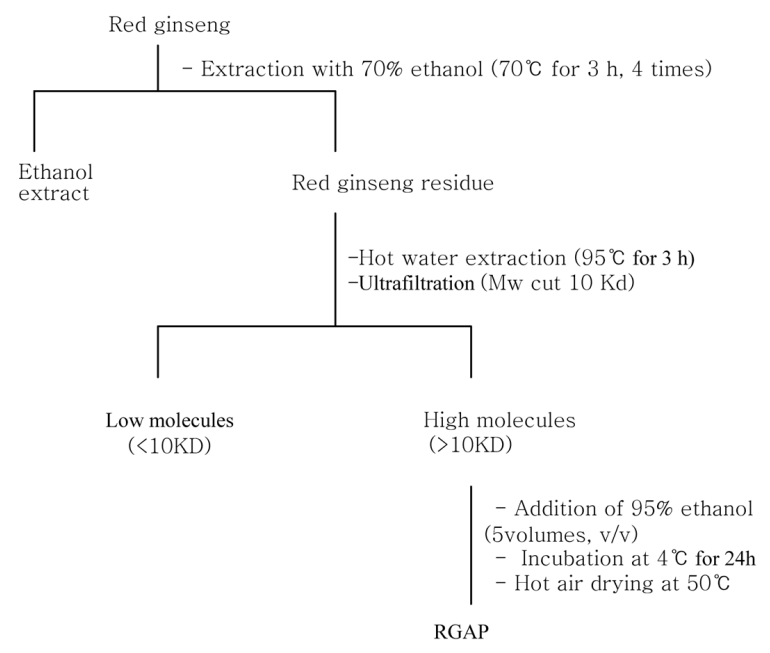

(B)

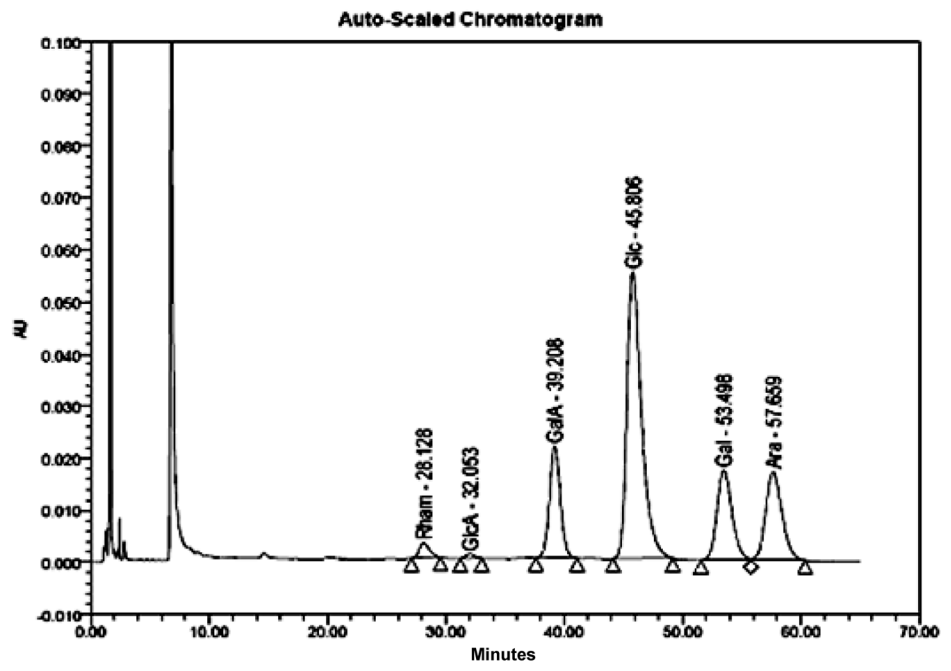

Fig. 1. Purification Procedure of RGAP and HPLC Profile of Its Component Sugars

(A) RGAP was prepared by $70 \%$ extraction, hot water extraction, and ultrafiltration. (B) The analysis of component sugars in RGAP was performed by HPLC with CAPCELL PAK C18 column using a mobile phase condition [0.1 m sodium phosphate buffer ( $\mathrm{pH} 7.0)$ 87: acetonitrile 13]. 
liver were enzymatically determined by assay kit (Asan Pharmacy, Korea).

LPL Activity Assay LPL activity was measured from plasma using an ELISA (Daichi Pure Chemical Company, Tokyo, Japan). The activity was expressed in units representing nmol of free fatty acids released per $\mathrm{ml}$ of plasma.

Reverse Transcription-Polymerase Chain Reaction (RT-PCR) For the evaluation of cytokine mRNA expression levels, total RNA from liver was prepared by adding TRIzol Reagent (Gibco BRL) according to the manufacturer's protocol. Semi-quantitative RT reactions were conducted using MuLV reverse transcriptase. The total RNA $(1 \mu \mathrm{g})$ was incubated with oligo-dT15 for $5 \mathrm{~min}$ at $70^{\circ} \mathrm{C}$ and mixed with a $5 \times$ first-strand buffer, $10 \mathrm{~mm}$ of deoxyribonucleotide triphosphate (dNTP), and $0.1 \mathrm{M}$ DTT. The reaction mixture was further incubated for $5 \mathrm{~min}$ at $37^{\circ} \mathrm{C}$ and for $60 \mathrm{~min}$ after the addition of $\mathrm{MuLV}$ reverse transcriptase (2 U). Reactions were terminated after $10 \mathrm{~min}$ at $70^{\circ} \mathrm{C}$, and the total RNA was depleted by adding RNase H. The PCR reaction was conducted with the incubation mixture $(2 \mu \mathrm{l}$ cDNA, $4 \mu \mathrm{M} 5^{\prime}$ and $3^{\prime}$ primers, a $10 \times$ buffer $[10 \mathrm{~mm}$ Tris- $\mathrm{HCl}, \mathrm{pH} 8.3,50 \mathrm{~mm} \mathrm{KCl,} \mathrm{0.1 \%} \mathrm{Triton} \mathrm{X-100],} 250 \mu \mathrm{M}$ of dNTP, $25 \mathrm{~mm}$ of $\mathrm{MgCl}_{2}$, and 1 unit of Taq polymerase [Promega, U.S.A.]). The following incubation conditions were used: a $45 \mathrm{~s}$ denaturation time at $94^{\circ} \mathrm{C}$, an annealing time of $45 \mathrm{~s}$ between 55 and $60^{\circ} \mathrm{C}$, an extension time of $60 \mathrm{~s}$ at $72{ }^{\circ} \mathrm{C}$, and final extension of $7 \mathrm{~min}$ at $72{ }^{\circ} \mathrm{C}$. The primers (Bioneer, Seoul, Korea) for LPL were located at nucleotides 1303 to 1322 , and 1589 to 1608 of the LPL cDNA, as reported previously. ${ }^{25)}$

Statistical Analysis Results were represented as mean \pm S.D. Statistical significance was determined by the paired student $t$-test. $p$ values less than 0.05 or 0.01 were considered significant.

\section{RESULTS AND DISCUSSION}

Polysaccharides isolated from basidiomycetes have been reported to act as both immunostimulator and anti-hyperlipi- demic activator. The fungal polysaccharides (e.g., lentinan) originating from Lentinus edodes composed of the basic structure of a $\beta$-1,3-glucan with $\beta$-1,6-glucopyranosidic branches also showed both immunostimulating properties and anti-hyperlipidemic activities. ${ }^{26-28)}$ In contrast, RGAP has only been found to have immunostimulating and antitumor activities in tumor-bearing models, ${ }^{11)}$ but no reports addressed its regulatory role in hyperlipidemia. As a part of a continuing investigation of RGAP, therefore, its antihyperlipidemic effects were evaluated using both Triton WR1339-induced endogenous and corn oil-induced exogenous hyperlipdemic rat models.

Our data indicate that RGAP was able to modulate hyperlipidemic conditions as effectively as other polysaccharides did. ${ }^{28)}$ Oral administration of RGAP (200 to $1000 \mathrm{mg} / \mathrm{kg}$ ) significantly reduced Triton WR1339-induced serum TG levels which had increased up to 3.59 times normal, in the same way as $100 \mathrm{mg} / \mathrm{kg}$ of gemfibrozil (Gem 100), an oral drug used to lower lipid levels (Fig. 2). ${ }^{29)}$ Administration of RGAP markedly reduced the up-regulated levels of NEFA, considered as a risk factor for atherosclerosis, ${ }^{30,31)}$ under Triton WR1339 treatment conditions (Fig. 2). On the contrary, unlike other polysaccharide-type modulators such as chitosan, $\beta$-glucans and hydroxypropylmethylcellulose, ${ }^{32-34)}$ the Triton WR1339-induced increase in TC concentration was not significantly affected by RGAP treatment, although there was an inhibitory tendency at higher doses $(500,1000 \mathrm{mg} / \mathrm{kg})$ (Fig. 2). Similarly, RGAP administration did not diminish the enhanced levels of phospholipids in serum during Triton WR1339 treatment (Fig. 2), indicating that the metabolisms of phospholipids as well as cholesterol might not be a target to be lowered by RGAP treatment. In agreement with serum levels, RGAP displayed similar suppressive patterns in the regulation of hepatic total lipid and TG (Table 1). That is, RGAP treated groups $(500,1000 \mathrm{mg} / \mathrm{kg})$ significantly lowered hepatic levels of total lipids and TG by normal levels. As in the case of serum parameters, however, there was no significant difference in the hepatic levels of TC and HDLcholesterol (HDL-C) between control and RGAP administra-
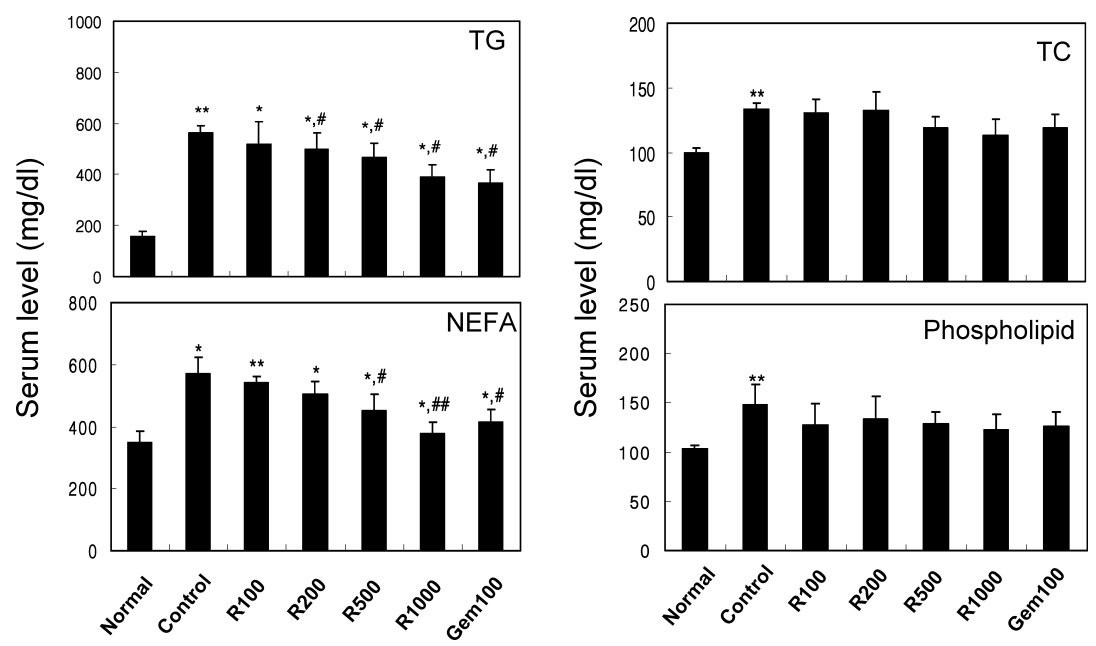

Fig. 2. Effect of RGAP on the Serum Level of TG, NEFA, TC, and Phospholipid in Endogenous Hyperlipidemic Rats Induced by Triton WR1339

Rats orally administered with RGAP for $3 \mathrm{~d}$ were intravenously injected with Triton WR1339 (150 mg/kg). Serum was prepared from blood drawn by heart puncture $2 \mathrm{~h}$ after final RGAP administration. Serum levels of TG, NEFA, TC and phospholipid were measured by the Automatic analyzer, as described in Materials and Methods. Data represent mean \pm S.E.M. of three independent observations performed with 10 rats. $* p<0.05$ and $* * p<0.01$ compared to normal group, $\# p<0.05$ and $\# p<0.01$ compared to control group (Triton WR1339 alone). 
Table 1. Effect of RGAP on the Levels of TC, TG and HDL-C in the Liver of Endogenos Hyperlipidemic Rats Induced by Triton WR1339

\begin{tabular}{lllll}
\hline \hline $\begin{array}{l}\text { Group } \\
(n=8)\end{array}$ & $\begin{array}{c}\text { Total lipid } \\
(\mathrm{mg} / \mathrm{g} \text { of tissue })\end{array}$ & $\begin{array}{c}\mathrm{TG} \\
(\mathrm{mg} / \mathrm{g})\end{array}$ & $\begin{array}{c}\mathrm{TC} \\
(\mathrm{mg} / \mathrm{g})\end{array}$ & $\begin{array}{c}\text { HDL-C } \\
(\mathrm{mg} / \mathrm{g})\end{array}$ \\
\hline NC & $23.4 \pm 0.95$ & $1.99 \pm 0.20$ & $0.43 \pm 0.03$ & $0.92 \pm 0.02$ \\
Control & $24.7 \pm 0.5^{*}$ & $2.22 \pm 0.20^{* *}$ & $0.49 \pm 0.02$ & $0.79 \pm 0.02^{*}$ \\
R100 & $23.3 \pm 0.68$ & $2.19 \pm 0.07$ & $0.48 \pm 0.03$ & $0.81 \pm 0.03$ \\
R500 & $22.6 \pm 0.43^{\#}$ & $2.01 \pm 0.27^{\#}$ & $0.47 \pm 0.02$ & $0.80 \pm 0.04$ \\
R1000 & $22.3 \pm 0.86^{\#}$ & $1.83 \pm 0.26^{\#}$ & $0.48 \pm 0.02$ & $0.79 \pm 0.03$
\end{tabular}

$* p<0.05$ and $* * p<0.01$ compared to normal group, ${ }^{*} p<0.05$ compared to control group (Triton WR1339 alone).

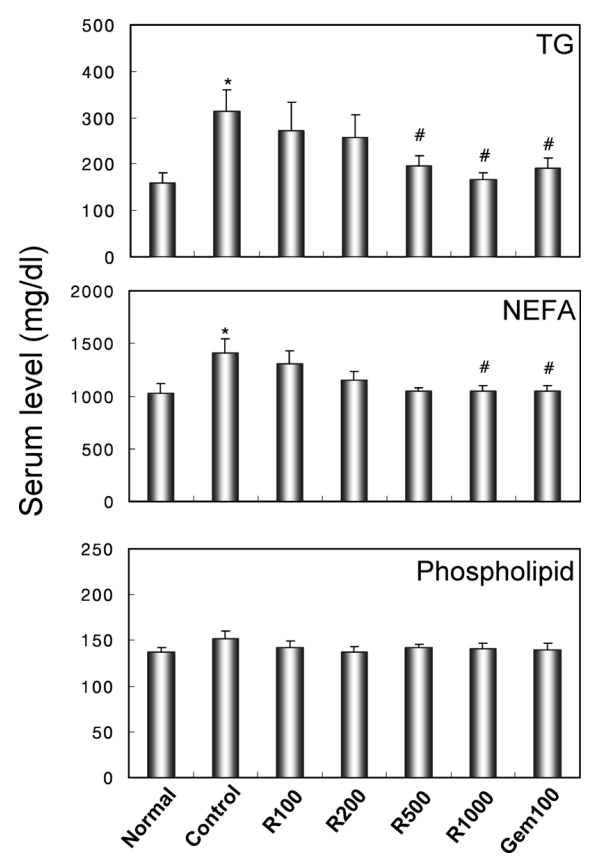

Fig. 3. Effect of RGAP on the Serum Level of TG, NEFA, and Phospholipid in Exogenous Hyperlipidemic Rats Induced by Corn Oil

Rats orally administered with RGAP for $3 \mathrm{~d}$ were intravenously injected with corn oil $(2 \mathrm{~g} / \mathrm{g})$. Serum was prepared from blood drawn by heart puncture $2 \mathrm{~h}$ after final RGAP administration. Serum levels of TG, NEFA, and phospholipid were measured by the Automatic analyzer, as described in Materials and Methods. Data represent mean \pm S.E.M. of three independent observations performed with 10 rats. $* p<0.05$ compared to normal group, ${ }^{\#} p<0.05$ and ${ }^{\#} p<0.01$ compared to control group (corn oil alone).

tion groups.

To confirm the regulatory effect of RGAP on endogenous hyperlipidemic conditions, a corn oil-induced exogenous model was tested. Corn oil treatment strikingly increased the serum levels of TG and NEFA (Fig. 3) by up to $83 \%$ and liver TG levels by up to $15 \%$ (Table 2), similar to previous reports. ${ }^{35)}$ Administration of $\operatorname{RGAP}(500,1000 \mathrm{mg} / \mathrm{kg})$ strongly reduced the serum TG levels in this model in a dose dependent manner. Enhanced serum level of NEFA was also diminished at 500 and $1000 \mathrm{mg} / \mathrm{kg}$ doses. In contrast, the level of serum phospholipids was not significantly reduced by RGAP treatment (Fig. 3). Similarly, the levels of TG and NEFA were lowered by $100 \mathrm{mg} / \mathrm{kg}$ of gemfibrozil (Gem 100) by up to $39.1 \%$. In exogenous corn oil model, the hepatic levels of TG were significantly down-regulated by the administration of RGAP at $500 \mathrm{mg} / \mathrm{kg}$. However, none of the other hepatic parameters displayed significant changes in the exogenous hyperlipidemic rat model (Table 2).

Finally, how RGAP can negatively modulate the serum
Table 2. Effect of RGAP on the Levels of TC, TG and HDL-C in the Liver of Exogenous Hyperlipidemic Rats Induced by Corn Oil

\begin{tabular}{lcccc}
\hline \hline $\begin{array}{c}\text { Group } \\
(n=8)\end{array}$ & $\begin{array}{c}\text { Total lipid } \\
(\mathrm{mg} / \mathrm{g} \text { of tissue })\end{array}$ & $\begin{array}{c}\mathrm{TG} \\
(\mathrm{mg} / \mathrm{g})\end{array}$ & $\begin{array}{c}\mathrm{TC} \\
(\mathrm{mg} / \mathrm{g})\end{array}$ & $\begin{array}{c}\text { HDL-C } \\
(\mathrm{mg} / \mathrm{g})\end{array}$ \\
\hline NC & $23.5 \pm 0.95$ & $1.89 \pm 0.30$ & $0.51 \pm 0.05$ & $0.88 \pm 0.03$ \\
Control & $23.7 \pm 0.5$ & $2.15 \pm 0.25^{*}$ & $0.56 \pm 0.03$ & $0.80 \pm 0.02$ \\
R100 & $23.3 \pm 0.7$ & $2.10 \pm 0.17$ & $0.54 \pm 0.04$ & $0.81 \pm 0.03$ \\
R300 & $23.6 \pm 0.6$ & $2.05 \pm 0.27$ & $0.54 \pm 0.03$ & $0.80 \pm 0.04$ \\
R500 & $23.3 \pm 0.6$ & $1.95 \pm 0.30^{\#}$ & $0.54 \pm 0.03$ & $0.85 \pm 0.04$ \\
\hline
\end{tabular}

$* p<0.05$ compared to normal group, ${ }^{*} p<0.05$ compared to control group (corn oil alone).

(A)

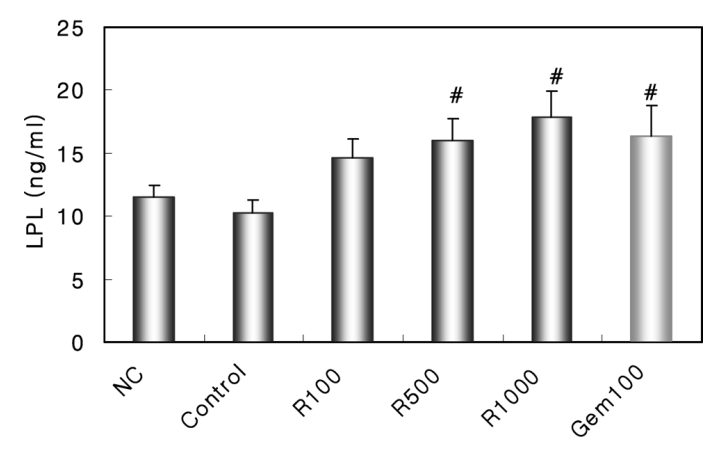

(B)

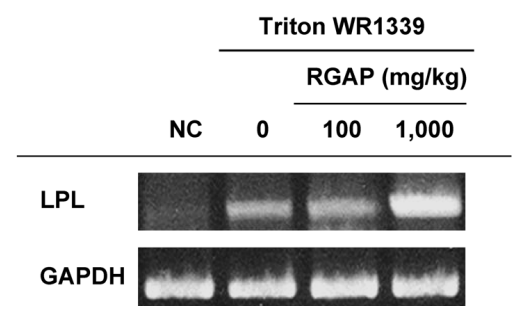

Fig. 4. Effect of RGAP on Serum LPL Activity in Hyperlipidemic Rats Induced by Triton WR1339

Rats orally administered with RGAP for $3 \mathrm{~d}$ were intravenously injected with Triton WR1339 $(150 \mathrm{mg} / \mathrm{kg})$. (A) Serum was prepared from blood drawn by heart puncture $2 \mathrm{~h}$ after final RGAP administration. Serum activity of LPL was measured by an ELISA method, as described in Materials and Methods. (B) mRNA level of LPL in liver of hyperlipidemic rats treated with RGAP was determined by RT-PCR. Data represent mean \pm S.E.M. of three independent observations performed with 10 rats. ${ }^{\#} p<0.05$ compared to control group (Triton WR1339 alone).

and hepatic levels of TG and NEFA but not cholesterol and phospholipids was further evaluated using TG hydrolysis levels. Since it is known that LPL is a key atheroprotective enzyme $^{36)}$ in regulating plasma TG levels by removing TG-rich lipoproteins from circulating plasma, ${ }^{19,37)}$ and that Triton WR1339 inhibits LPL activity in serum by increasing TG levels ${ }^{38)}$ we carefully tested whether RGAP is able to modulate LPL to explain its anti-hyperlipidemic activity with hypotriglyceridemic action. Interestingly, Fig. 4A depicts that Triton WR1339 treatment lowered LPL activity compared to normal, although it was not statistically significant. Orally administered RGAP dose-dependently up-regulated LPL activity up to $75.3 \%$ (Fig. 4A), in ways similar to other polysaccharides such as Auricularia auricular-derived polysaccharides, fucoidan. ${ }^{37,39)}$ Therefore, these results suggest that 
treatment with RGAP in rats may modulate hyperlipidemic conditions via up-regulating the degradation enzyme (e.g., LPL) activity of lipoprotein. Whether this enhancement of LPL activity was linked to its up-regulation of LPL expression level was continuously examined. Interestingly, 1000 $\mathrm{mg} / \mathrm{kg}$ of RGAP remarkably increased the mRNA level of LDL (Fig. 4B), suggesting that enhanced expression of LPL seems to mediate its up-regulated activity. However, because Triton WR1339 also increased the mRNA level of LPL compared to normal condition, unlike its down-regulated activity (Fig. 4), evaluation using mRNA level itself seems not to be crucial until there is a big difference in its expression level. Otherwise, other factors seem to be involved in the regulation of LPL activity. Indeed, the fact that the binding between LPL and heparan sulfate proteoglycans plays a critical role in LPL function ${ }^{40)}$ seems to suggest another possibility that a direct interaction between LPL and RGAP can be an important biochemical event. To prove this possibility, further analysis will be carried out in the following experiment.

In summary, we found that RGAP treatment reduced both the levels of TG in serum and the liver and increased the activity of LPL, in serum, indicating that RGAP may diminish the levels of TG through activation of LPL. Considering that the higher molecular weight $\beta$-glucan is clinically effective in controlling blood lipid composition ${ }^{41)}$ and $45 \%$ of red ginseng extract is acid polysaccharide portion, it is suggested that the anti-hyperlipidemic activity of RGAP or red ginseng extract seems to be available in the human body, although its anti-hyperlipidemic efficacy was seen at higher doses. Whether RGAP can be developed to be a functional food with anti-hyperlipidemic properties will be examined in the next clinical trial. Moreover, what component of RGAP is responsible factor for its anti-hyperlipidemic effects should be also followed to reach more detailed pharmacological understanding.

Acknowledgment This work was supported by a grant from Korean Ginseng Corporation (KGC).

\section{REFERENCES}

1) Blumenthal M., Adv. Nurse Pract., 9, 26-28, 33 (2001).

2) Choi K. T., Acta Pharmacol. Sin., 29, 1109-1118 (2008).

3) Park T. Y., Park M. H., Shin W. C., Rhee M. H., Seo D. W., Cho J. Y., Kim H. M., Biol. Pharm. Bull., 31, 1802-1805 (2008).

4) Min S. W., Jung S. H., Cho K. H., Kim D. H., Biomol. Therapeut., 16, 364-369 (2008).

5) Park T. Y., Cho J. Y., Biomol. Therapeut., 16, 370-376 (2008).

6) Lee J. H., Jeong C. S., Biomol. Therapeut., 16, $277-285$ (2008).

7) Konno C., Sugiyama K., Kano M., Takahashi M., Hikino H., Planta Med., 50, 434-436 (1984)

8) Oshima Y., Konno C., Hikino H., J. Ethnopharmacol., 14, 255-259 (1985).

9) Konno C., Murakami M., Oshima Y., Hikino H., J. Ethnopharmacol., 14, 69-74 (1985).
10) Park K. M., Kim Y. S., Jeong T. C., Joe C. O., Shin H. J., Lee Y. H., Nam K. Y., Park J. D., Planta Med., 67, 122-126 (2001).

11) Du X. F., Jiang C. Z., Wu C. F., Won E. K., Choung S. Y., Arch. Pharm. Res., 31, 1153-1159 (2008).

12) Lee Y. S., Chung I. S., Lee I. R., Kim K. H., Hong W. S., Yun Y. S., Anticancer Res., 17, 323-331 (1997).

13) Takaku T., Kameda K., Matsuura Y., Sekiya K., Okuda H., Planta Med., 56, 27-30 (1990).

14) Yoo B. C., Park G. H., Okuda H., Takaku T., Kim S., Hwang W. I., Amino Acids, 17, 391-400 (1999).

15) Goldfarb S., J. Lipid Res., 19, 489-494 (1978).

16) Borensztajn J., Rone M. S., Kotlar T. J., Biochem. J., 156, 539-543 (1976).

17) Duhault J., Boulanger M., Beregi L., Sicot N., Bouvier F., Atherosclerosis, 23, 63-72 (1976).

18) Shin H. J., Kim Young So., Kwak Y. S., Song Y. B., Kim Young Sa., Park J. D., Planta Med., 70, 1033-1038 (2004).

19) Phillips C., Owens D., Collins P., Tomkin G. H., Atherosclerosis, 181, 109-114 (2005).

20) Hofbauer B., Friess H., Weber A., Baczako K., Kisling P., Schilling M., Uhl W., Dervenis C., Buchler M. W., Gut, 38, 753-758 (1996).

21) Takahashi Y., Inaba N., Kuwahara S., Kuki W., Biosci. Biotechnol. Biochem., 67, 2448-2450 (2003).

22) Parker R. A., Sabrah T., Cap M., Gill B. T., Arterioscler. Thromb. Vasc. Biol., 15, 349-358 (1995).

23) Park J. D., Song Y. B., Kwak Y. S., Kim J. C., Im D. H., Han J., J. Toxicol. Pub. Health, 19, 173-180 (2003).

24) Ametaj B. N., Bobe G., Lu Y., Young J. W., Beitz D. C., J. Agric. Food Chem., 51, 2105-2110 (2003).

25) Ong J. M., Simsolo R. B., Saghizadeh M., Pauer A., Kern P. A., J. Lipid Res., 35, 1542-1551 (1994).

26) Franz G., Planta Med., 55, $493-497$ (1989).

27) Lee J. Y., Kim J. Y., Lee Y. G., Rhee M. H., Hong E. K., Cho J. Y., J. Microbiol. Biotechnol., 18, 355-364 (2008).

28) Soeda S., Ohmagari Y., Shimeno H., Nagamatsu A., Biol. Pharm. Bull., 17, 784-788 (1994).

29) Roy A., Pahan K., Immunopharmacol. Immunotoxicol., 31, 339-351 (2009).

30) Shafrir E., J. Clin. Invest., 37, 1775-1782 (1958).

31) Lloyd E. E., Gaubatz J. W., Burns A. R., Pownall H. J., Atherosclerosis, 192, 49-55 (2007).

32) Zhang J., Liu J., Li L., Xia W., Nutr. Res., 28, 383-390 (2008).

33) Reppas C., Swidan S. Z., Tobey S. W., Turowski M., Dressman J. B., Eur. J. Clin. Nutr., 63, 71-77 (2009).

34) Wilson T. A., Nicolosi R. J., Delaney B., Chadwell K., Moolchandani V., Kotyla T., Ponduru S., Zheng G. H., Hess R., Knutson N., Curry L., Kolberg L., Goulson M., Ostergren K., J. Nutr., 134, 2617-2622 (2004).

35) MacDonald R. S., Zhang W., Zhang J. P., Sun G. Y., J. Nutr., 126, 1554-1562 (1996)

36) Chiba T., Miura S., Sawamura F., Uetsuka R., Tomita I., Inoue Y., Tsutsumi K., Tomita T., Arterioscler. Thromb. Vasc. Biol., 17, 26012608 (1997).

37) Yokota T., Nagashima M., Ghazizadeh M., Kawanami O., Life Sci., 84, 523-529 (2009).

38) Millar J. S., Cromley D. A., McCoy M. G., Rader D. J., Billheimer J. T., J. Lipid Res., 46, 2023 -2028 (2005).

39) Chen G., Luo Y. C., Li B. P., Li B., Guo Y., Li Y., Su W., Xiao Z. L., J. Food Sci., 73, H103-H108 (2008).

40) Spillmann D., Lookene A., Olivecrona G., J. Biol. Chem., 281, 23405-23413 (2006).

41) Keenan J. M., Goulson M., Shamliyan T., Knutson N., Kolberg L., Curry L., Br. J. Nutr., 97, 1162-1168 (2007). 